\title{
If you don't want them shed them
}

\author{
Cell Research (2013) 23:171-172. doi:10.1038/cr.2012.121; published online 21 August 2012
}

Seminal studies in C. elegans contributed to our general understanding of programmed cell death conferred by apoptosis. A recent study unravelled a new form of cell death in the worm and provided insights into its regulation. Affected cells are shed from intact tissues, a modality of death likely to be conserved and relevant to cancer.

At a first glance metazoan development and tissue homeostasis appears as rather wasteful processes. Superfluous and unwanted cells are generated, but are then eliminated by cell death. Cell death can come in various forms and studies on the roundworm C. elegans provided important insights. Taking the extremes cells might be merely killed by necrosis, a process, which at the first superficial glance might not even involve an active cellular process. Generally, however, cell death appears to be programmed, and in most cases is regulated akin to cellular suicide. Studies on C. elegans were instrumental to define apoptosis, a form of programmed cell death now generally referred to be dependent on caspases. The activation of these proteases, which trigger the cellular demise, generally appears as the last step of activating the conserved apoptosis pathway. Kerr and Wyllie first described 'apoptosis' in mammalian tissues, and their description at the time was solely based on morphological features of 'apoptotic' cells [1]. Features include chromatin compaction, cellular shrinkage and membrane blebbing. Apoptosis also became apparent when the near perfectly invariant development of the nematode worm C. elegans was studied; 131 cells of the 1090 cells, which are born, are eliminated and the majority of those cells show the hallmark features of apoptosis [2]. Starting by looking for mutants with reduced or excessive apoptosis, the major players of the apoptotic pathway were defined. The BH3 only domain protein EGL-1 inhibits the conserved anti-apoptotic CED-9/Bcl2 cell survival protein $[3,4]$. Such inhibition leads to the activation of pro-apoptotic CED-4, a protein related to mammalian Apaf-1 and likewise required for caspase activation [5-7]. In the worm, the sole major pro-apoptotic caspase is CED-3.

A recent Nature paper by Denning et al. [8] describes a novel form of developmentally regulated programed cell death in $C$. elegans. This cell death, which the authors refer to as cell shedding, is independent of the CED-3 caspase as well as of the three further caspases encoded in the $C$. elegans genome. What the authors observed is that cells are extruded from the embryo during embryogenesis. These cells often occur in groups and remain enclosed in the eggshell during embryonic development. Otherwise the morphology of those cells, the generation of which does not require any known C. elegans apoptosis proteins, appears to resemble genuine apoptotic cells. Chromatin condensation and the separation of the nuclear envelope double membrane occur, and DNA fragmentation becomes obvious upon TUNEL staining. Why were those deaths not found and followed up at an earlier stage? Cell shedding, at least in the worm, appears to be a process that occurs redundantly with the general apoptosis pathway. Only when apoptosis is blocked, cell shedding becomes obvious as the eight cells that can be eliminated by shedding normally die by apoptosis. Thus shedding can only be observed in apoptosis-defective mutants. Recording the development of apoptosis-defective mutants and tracing back the developmental origin of "shedded' cells, it became apparent that those cells are the very same cells that are the first to normally die by apoptosis. It had been known that apoptotic cells, which fail to be engulfed at times, can be separated from the embryo [9]. However, these 'undead' cells, which the authors refer to as floaters, are morphologically distinct to shedded cells.

Denning et al. [8] went further to find the regulators of the death, which is conferred by shedding. Again the information provided by the invariant developmental lineage, and the ability to study development at the cellular level came to their aide. The authors managed to determine the cell identity of one of the shed cells. When a cell fails to be dismantled by apoptosis, it often takes the fate of its sister cell. The sister of one of the cells, which can be eliminated by apoptosis or shedding, is fated to produce the excretory cell [10]. This cell differentiates to act as the kidney of the worm, and appropriate 'tissue' specific GFP markers are known. All the authors had to do, was to look for mutants where, in the absence of apoptosis, the sister cell would not be shed and an additional excretory cells would occur. The first such mutant that they defined was pig-1, the C. elegans counterpart of mammalian MELK, a member of the AMPK-related serine threonine protein kinase family. PIG-1 regulates the asymmetric division of 
C. elegans neuroblasts, and mammalian AMPK-related kinases control cell metabolism and polarity [11]. AMPK kinases are activated via the phosphorylation of their T-loop domain by the LKB1/PAR-4 kinase, which acts in complex with STRAD $\alpha$ and MO25 $\alpha$ [12]. Intriguingly, human LKB1 mutations cause Peutz-Jeghers syndrome, an autosomal dominantly inherited disorder; the pathology of this syndrome includes intestinal polyps linked to excessive intestinal cells [13]. Double mutants of ced-3 with components of the worm LKB1 complex phenocopied the ced-3 pig-1 phenotype, equally leading to a reduced number of shedded cells and the ectopic generation of an additional excretory cell. Double mutant analysis and the finding that PIG-1 T-loop phosphorylation is essential for shedding confirmed that PIG-1/LKB1 acts in a linear kinase pathway needed for shedding.

The next step was to ask how these kinases might act to affect shedding. It is reasonable to postulate that components of cell-to-cell adhesion complexes might be involved in the shedding process. The authors therefore tested whether HMP1 , a C. elegans $\alpha$-catenin, is expressed in shedded cells using an appropriate GFP fusion. While HMP-1::GFP is expressed at the surface of cells next to the cell fated to be shed, cells that were shed did not show such expression. In contrast, HMP-1::GFP was expressed in cells that are fated to shed, but fail to be shedded in pig-1 ced-3 double mutants. Thus, it is reasonable to postulate that PIG-1 can modulate cell shedding by supressing the appropriate expression of cell adhesion molecules.

These results are highly relevant. While cryptic death in wild-type worms by shedding might nevertheless represent a general and evolutionary conserved programmed cell death pathway. It had been previously reported that cells can be shed from epithelial cell layers [14]. Intriguingly, polyposis associated with Peutz-Jeghers syndrome might well not be solely associated with the excessive proliferation of gut epithelia, but also be linked to the reduced shedding. It will be interesting to see whether the same mechanisms uncovered in the worm also act in this or mammalian developmental systems, and whether they are relevant to cancer. Cell death by shedding adds to the repertoire of cell death mechanisms uncovered in the worm and is likely to be generally important. Previous studies already indicated that specific, non-caspase related proteases alleviated necrotic death, and studies on the demise of a single cell, the male 'linker cell', reveal an non-apoptotic death program, the morphological features of which equally appear to be conserved [15]. All in all these are exciting studies.
Ehsan Pourkarimi ${ }^{1}$, Anton Gartner ${ }^{1}$

${ }^{l}$ Wellcome Trust Centre for Gene Regulation and Expression, University of Dundee, Dundee DD1 5EH, UK

Correspondence: Anton Gartner

E-mail: a.gartner@dundee.ac.uk

\section{References}

1 Kerr JF, Wyllie AH, Currie AR. Br J Cancer 1972; 26:239-257.

2 Sulston JE, Schierenberg E, White JG, et al. Dev Biol 1983; 100:64-119.

3 Conradt B, Horvitz HR. Cell 1998; 93:519529.

4 Hengartner MO, Ellis RE, Horvitz HR. Nature 1992; 356:494-499.

5 Ellis HM, Horvitz HR. Cell 1986; 44:817829.

6 Yuan JY, Horvitz HR. Dev Biol 1990; 138:33-41.

7 Yuan J, Horvitz HR. Development 1992; 116:309-320.

8 Denning DP, Hatch V, Horvitz HR. Nature 2012; 488:226-230.

9 Wu YC, Stanfield GM, Horvitz HR. Genes Dev 2000; 14:536-548.

10 Buechner M. Trends Cell Biol 2002; 12:479484.

11 Cordes S, Frank CA, Garriga G. Development 2006; 133:2747-2756.

12 Lizcano JM, Göransson $\mathrm{O}$, Toth $\mathrm{R}$, et al. EMBO J 2004; 23:833-843.

13 Hemminki A, Avizienyte E, Roth S, et al. Duodecim 1998; 114:667-668.

14 Rosenblatt J, Raff MC, Cramer LP. Curr Biol 2001; 11:1847-1857.

15 Abraham MC, Lu Y, Shaham S. Dev Cell 2007; 12:73-86. 